\title{
Efeito da Freqüência de Pastejo e da Época do Ano sobre a Produção e a Qualidade em Panicum maximum cvs. Tanzânia e Mombaça
}

\author{
Patrícia Menezes Santos ${ }^{1}$, Moacyr Corsi ${ }^{2}$, Marco Antônio Alvares Balsalobre ${ }^{1}$
}

\begin{abstract}
RESUMO - Foram realizados dois experimentos para avaliar o efeito de três freqüências de pastejos (28, 38 e 48 dias) sobre a massa de forragem, a taxa de acúmulo de matéria seca (MS) e a relação folha:haste (F:H) em Tanzânia e Mombaça, no período de setembro de 1995 a maio de 1996. O delineamento experimental foi em parcelas subdivididas, sendo a parcela representada pela freqüência de pastejo e as sub-parcelas pelos períodos do ano. A massa de forragem por pastejo foi maior quando a freqüência de pastejo foi menor, porém não teve efeito sobre a taxa de acúmulo de MS nos cultivares Tanzânia e Mombaça. A taxa de acúmulo de matéria seca para os dois cultivares foi maior em janeiro/fevereiro; portanto, nesta época, deve-se trabalhar com freqüências de pastejo ou lotações mais elevadas. A relação folha:haste dos dois cultivares foi baixa, principalmente durante a fase reprodutiva, indicando que se deve trabalhar com freqüências de pastejo mais elevadas (pastejos a cada 28 dias ou menos), a fim de controlar o desenvolvimento das hastes.
\end{abstract}

Palavras-chave: massa de forragem, relação folha:haste, taxa de acúmulo de matéria seca

\section{Effects of Grazing Frequency and Season of the Year on Yield and Quality of Panicum maximum cvs. Tanzania e Mombaça}

\begin{abstract}
Two experiments were carried out to evaluate the effects of three grazing frequencies (28, 38 and 48 days) on forage mass, dry matter(DM) accumulation rate and leaf:stem ratio in Tanzania and Mombaça, from September 1995 to May 1996 period. A split plot experimental design was used, with grazing frequency as main plots and period of the year as subplots. The forage mass per grazing was greater when the grazing frequency was small, however did not have an effect on the DM accumulation in the Tanzania and Mombaça cultivars. The rates of forage accumulation for the two cultivars were greater in January/February; so we should work with greater grazing frequency or stocking rate. The leaf:stem ratio of the two cultivars was small, mainly during the reproductive phase, indicating that we should work with greater grazing frequency (grazing at each 28 days or less) in order to control the stems development.
\end{abstract}

Key Words: forage mass, leaf:stem ratio, dry matter accumulation rate

\section{Introdução}

As cultivares Tanzânia e Mombaça da espécie Panicum maximum foram lançadas comercialmente em 1990 e 1993, respectivamente, pelo Centro Nacional de Pesquisa de Gado de Corte (CNPGC) da EMBRAPA. No entanto, suas características ainda são pouco conhecidas e mais estudos são necessários para melhor determinar suas exigências quanto ao manejo e à fertilidade de solo.

O potencial forrageiro dessas cultivares pode ser verificado por meio dos resultados obtidos durante a avaliação dos acessos no CNPGC. A cultivar Mombaça produziu $41 \mathrm{t} / \mathrm{ha}$. ano de MS, apresentando, em média, $81,9 \%$ de folhas, $13,4 \%$ de proteína bruta nas folhas e 9,7\% nos colmos. Já a cultivar Tanzânia produziu $33 \mathrm{t} / \mathrm{ha}$.ano de MS e teve, em média, $80 \%$ de folhas, $12,7 \%$ de proteína bruta nas folhas e $9 \%$ nos colmos (SAVIDAN et al., 1990; JANK et al., 1994; e JANK, 1995).

Para a espécie Panicum maximum, sistemas rotacionados de pastejo são os mais indicados, principalmente devido às suas características de perfilhamento e à sua alta produtividade (CORSI, 1984). Dessa forma, torna-se importante o estudo do efeito dos períodos de ocupação e descanso sobre as características das plantas.

Experimentos realizados com diversas gramíneas forrageiras, inclusive o Panicum maximum, mostram que a redução da freqüência de cortes acarreta maior produção, porém com queda de qualidade (HASSAN et al., 1990; SINGH, 1995). A intensidade desses efeitos, no entanto, depende das características da espécie, da cultivar em questão e da época do ano.

A estacionalidade de produção na espécie Panicum maximum é um fato bem conhecido (JANK

${ }^{1}$ Mestre em Agronomia pela ESALQ/USP. Bolsista da CAPES.

2 Professor Titular do Departamento de Zootecnia da ESALQ/USP. 
SANTOS et al.

et al., 1994). Porém, poucos trabalhos procuram estudar as diferenças de produtividade ao longo da estação de crescimento (outubro a maio). A disponibilidade de forragem nesse período varia quando as plantas são submetidas a freqüências fixas de pastejo (COSTA et al., 1992). CECATO et al. (1996), trabalhando com oito cultivares de $P$. maximum cortadas a cada 35 dias, verificaram que a maior produção média por corte ocorreu em janeiro (10.008 kg MS/ha) e a menor, em abril (4521 kgMS/ha).

Dessa forma, em sistemas de produção nos quais se deseja trabalhar com alta eficiência de utilização da planta forrageira deve-se adotar freqüências de pastejo ou lotações variáveis ao longo da estação de crescimento. Em pastagens de azevém, por exemplo, a freqüência de pastejo é determinada com base na taxa de acúmulo líquido de forragem (PARSONS e PENNING, 1988).

O objetivo deste experimento foi avaliar o efeito de três freqüências de pastejo (28, 38 e 48 dias) sobre a massa de forragem, a taxa de acúmulo de MS e a relação F:H das cultivares Tanzânia e Mombaça durante o "verão" (outubro a maio).

\section{Material e Métodos}

Os experimentos foram conduzidos no Departamento de Zootecnia da ESALQ/USP, em PiracicabaSP (22042'30' 'S e 47038'30' 'W), no período de setembro de 1995 a maio de 1996 . O solo do local é classificado como terra roxa estruturada com as seguintes características químicas: $\mathrm{pH}$ em $\mathrm{CaCl}_{2}=$
5,8 ; matéria orgânica $=27 \mathrm{~g} / \mathrm{dm}^{3} ; \mathrm{P}$ (resina) $=$ $73 \mathrm{mg} / \mathrm{dm}^{3} ; \mathrm{S}=36,6 \mathrm{mg} / \mathrm{cm}^{3} ; \mathrm{K}=11,8 \mathrm{mmolc} / \mathrm{dm}^{3}$; $\mathrm{Ca}=73,5 \mathrm{mmolc} / \mathrm{dm}^{3} ; \mathrm{Mg}=29,8 \mathrm{mmolc} / \mathrm{dm}^{3}$; $\mathrm{H}+\mathrm{Al}=19,5 \mathrm{mmolc} / \mathrm{dm}^{3} ; \mathrm{Al}=0 \mathrm{mmolc} / \mathrm{dm}^{3} ; \mathrm{CTC}$ $=134,5 \mathrm{mmolc} / \mathrm{dm}^{3}$; e $\mathrm{V}=85,5 \%$. As cultivares Tanzânia e Mombaça foram plantadas em dezembro de 1994, em área de, aproximadamente, $5500 \mathrm{~m}^{2}$. Os dados de precipitação pluviométrica e temperatura encontram-se na Figura 1.

O delineamento experimental foi em blocos completos ao acaso com parcelas subdivididas no tempo e sete repetições. Para cada cultivar foi testado o efeito de três freqüências de pastejo (28,38 e 48 dias) e de quatro épocas do ano. Para as variáveis massa de forragem e relação $\mathrm{F}: \mathrm{H}$, os períodos considerados foram: 14/11/1995 a 31/12/1995 (novembro/dezembro), 1/1/1996 a 17/2/1996 (janeiro/fevereiro), 18/2/1996 a 5/4/1996 (fevereiro/abril) e 6/4/1996 a 23/5/1996 (abril/maio). A taxa de acúmulo de MS foi determinada nas seguintes épocas: 27/9 a 13/11/1995 (setembro/novembro), 14/11/1995 a 31/12/1995 (novembro/dezembro), 1/1/1996 a 17/2/1996 (janeiro/ fevereiro) e 18/2/1996 a 5/4/1996 (fevereiro/abril).

A área experimental foi dividida em 42 parcelas de $130 \mathrm{~m}^{2}$ (21 parcelas de cada cultivar). Foi realizado um corte de igualação em setembro de 1995. A partir daí, as parcelas foram pastejadas a cada 28,38 ou 48 dias, conforme o tratamento. A retirada dos animais das parcelas baseou-se na avaliação visual do resíduo pós pastejo, que foi, em média, 2300 e $1900 \mathrm{~kg}$

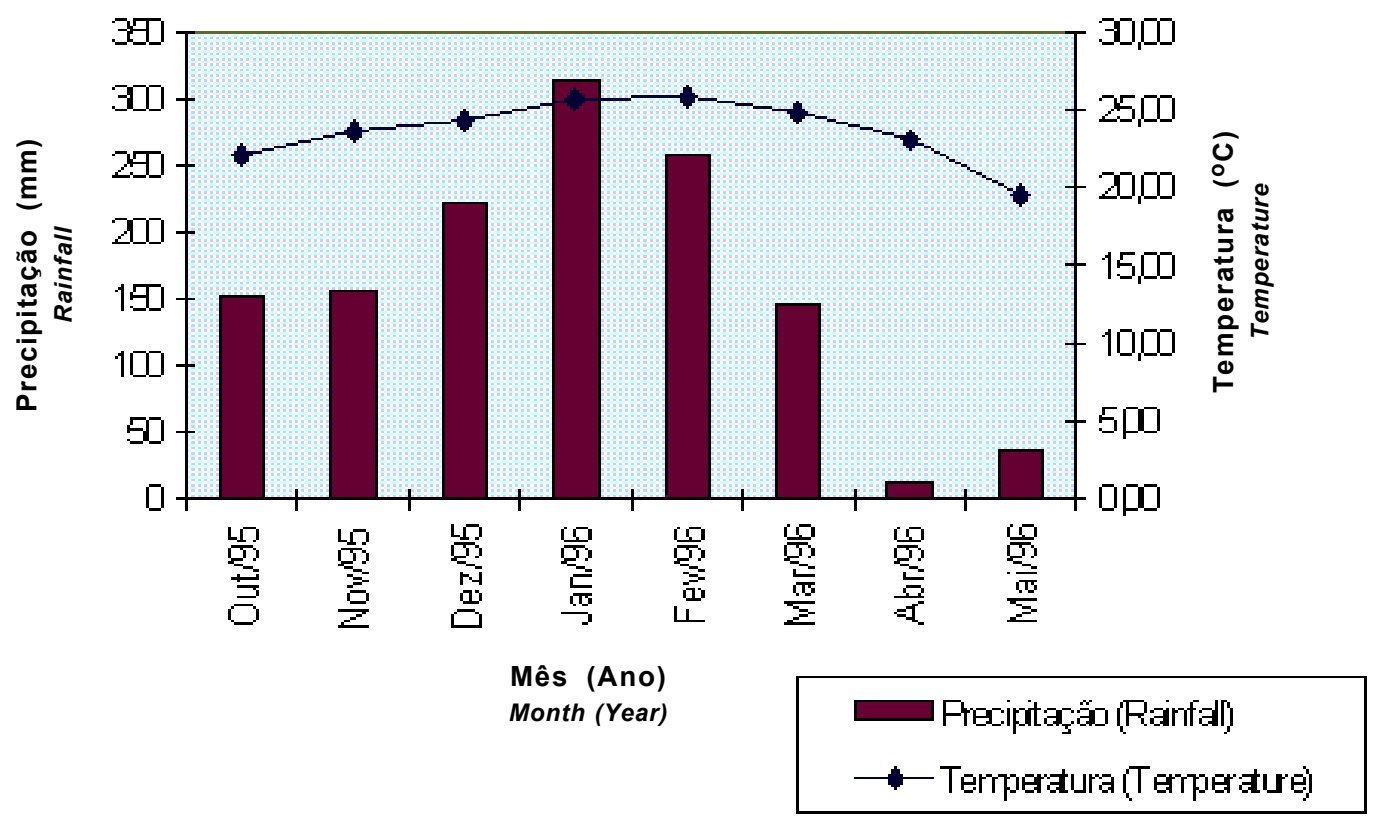

Figura 1 - Precipitação pluviomérica $(\mathrm{mm})$ e temperatura média $\left({ }^{\circ} \mathrm{C}\right)$ no período de setembro de 1995 a maio de 1996.

Figure 1 - Mean temperature and rainfall from September to May. 
246 Rev. bras. zootec.

MS/ha para as cultivares Mombaça e Tanzânia, respectivamente. Cada pastejo durou cerca de dois dias e foram utilizados entre 30 e 40 animais (vacas secas e novilhas da raça holandesa preto e branco).

As adubações foram feitas sempre após a saída dos animais do piquete. Todos os tratamentos receberam $400 \mathrm{~kg} / \mathrm{ha}$ de nitrogênio na forma de uréia, divididos em 6, 5 e 4 aplicações para 28, 38 e 48 dias de intervalo de pastejos, respectivamente.

Os parâmetros avaliados foram: massa de forragem; taxa de acúmulo de MS; e relação F:H.

Antes e após cada pastejo, foram cortadas, em cada parcela, quatro subamostras de $0,5 \mathrm{~m}^{2}(1,0 \mathrm{mx} 0,5 \mathrm{~m})$ no nível do solo. Após a pesagem no laboratório, as subamostras foram misturadas e uma amostra composta foi retirada, para se determinar o teor de $\mathrm{MS}\left(55^{\circ} \mathrm{C}\right)$ e a relação F:H. A massa de forragem em cada parcela foi a média das quatro subamostras e a relação $\mathrm{F}: \mathrm{H}$ foi calculada com base no peso seco. O cálculo da taxa de acúmulo de MS foi efetuado subtraindo-se da massa de forragem ao início de cada pastejo o resíduo do pastejo anterior e dividindo-se pelo intervalo de pastejos (dias).

Os dados de massa de forragem para as duas cultivares e de relação F:H para a cultivar Mombaça foram analisados segundo a transformação logarítmica decimal, enquanto os de relação $\mathrm{F}: \mathrm{H}$ da cultivar Tanzânia foram transformados usando-se a raiz quadrada recíproca. $\mathrm{O}$ modelo matemático adotado foi:

$$
Y_{i j k}=m+g_{i}+b j+e_{i j}+p_{k}+g p_{i k}+e_{i j k},
$$

em que

$Y_{i j k}=$ observação da freqüência de pastejos i, no bloco $\mathrm{j}$, na época do ano $\mathrm{k}$;

$\mathrm{m}=$ média da população;

$\mathrm{g}_{\mathrm{i}}=$ efeito da freqüência de pastejos $\mathrm{i}$;

$\mathrm{bj}=$ efeito do bloco $\mathrm{j}$;

$\mathrm{e}_{\mathrm{ij}}=$ efeito da variação do acaso

$\mathrm{p}_{\mathrm{k}}=$ efeito do época do ano $\mathrm{k}$.

$\mathrm{gp}_{\mathrm{ik}}=$ efeito da interação entre a freqüência de pastejos i e a época do ano k; e

$\mathrm{e}_{\mathrm{ijk}}=$ efeito da variação do acaso.

Os dados foram submetidos à análise de variância e as médias comparadas por intermédio do teste LSD ("Least significant difference"), utilizando-se os recursos do pacote estatístico SAS (1985).

\section{Resultados e Discussão}

A análise da variância não mostrou significância $(\mathrm{P}>0,05)$ para interação entre freqüência de pastejo e época do ano para a variável massa de forragem
Tabela 1 - Efeito da freqüência de pastejo sobre a massa de forragem por pastejo ( $\mathrm{kg} / \mathrm{ha})$ da cultivar Mombaça $^{1,2}$

Table 1 - Effect of grazing frequency on herbage mass $(\mathrm{kg} / \mathrm{ha})$ of Mombaça 1,2

\begin{tabular}{|c|c|}
\hline $\begin{array}{l}\text { Freqüência de } \\
\text { pastejo (dias) }\end{array}$ & $\begin{array}{l}\text { Massa de forragem } \\
\text { por pastejo }(\mathrm{kg} / \mathrm{ha})\end{array}$ \\
\hline$\frac{\text { Grazing frequency (days) }}{28}$ & $\frac{\text { Herbage mass }(\mathrm{kg} / \mathrm{ha})}{5731^{\mathrm{c}}}$ \\
\hline 38 & $7999^{b}$ \\
\hline 48 & $8904^{a}$ \\
\hline $\begin{array}{l}1 \text { Médias seguidas da mesn } \\
\text { pelo teste LSD }(P<0,10) \text {. } \\
2 \text { Os dados foram analisado } \\
\text { decimal. As médias foram } \\
{ }^{1} \text { Means followed by the same le } \\
(P<.10) \text {. } \\
2 \text { Herbage mass data are } \log _{10}\end{array}$ & $\begin{array}{l}\text { liferem significativamente } \\
\text { ransformação logarítmica } \\
\text { nadas. } \\
\text { er significantly by the } L S D \text { test } \\
\text { leans are back transformed }\end{array}$ \\
\hline
\end{tabular}

das duas cultivares logo, apenas os efeitos principais foram analisados.

A massa de forragem aumentou com o intervalo de pastejos, tanto na cultivar Tanzânia quanto na Mombaça (Tabela 1). CECATO et al. (1994), trabalhando com $P$. maximum cv. Aruana, observaram que o aumento do intervalo de cortes de 28 para 35 dias acarretou em maior produção de matéria seca, sendo que não houve diferença entre cortes aos 35, 42 e 49 dias. Já SINGH (1995) avaliando seis genótipos de $P$. maximum observou que a produção de MS aumentou quando o intervalo de cortes passou de 20 para 30 e para 40 dias. No caso das cultivares Mombaça e Tanzânia, houve diferença entre os intervalos de 38 e 48 dias, porém o aumento de produção foi mais expressivo quando se passou de 28 para 38 dias.

É importante observar que estes valores correspondem à massa de forragem por pastejo, pois, se for considerado que com pastejos mais freqüentes ocorre maior número de colheitas, a vantagem de aumentar o intervalo de pastejos torna-se menos significativa. Este fato pode ser avaliado por intermédio da taxa de acúmulo diário. Neste experimento, não houve efeito da freqüência de pastejo sobre a taxa de acúmulo de MS, ou seja, em termos de acúmulo total de forragem ao longo de toda a estação de crescimento, não há vantagem em reduzir a freqüência de pastejo.

A massa de forragem, para as duas cultivares, foi maior em janeiro/fevereiro, não havendo diferença estatística entre os demais períodos (Tabelas 1 e 2), o que pode ser conseqüência das condições extremamente favoráveis de temperatura e precipitação nessa época do ano (Figura 1). CECATO et al. (1996) também obtiveram produções mais elevadas para as 
248 Rev. bras. zootec.

Tabela 5 - Efeito da freqüência de pastejo sobre a relação folha:haste da cultivar Mombaça1,2

Table 5 - Effect of grazing frequency on leaf:stem ratio of Mombaça ${ }^{1,2}$

\begin{tabular}{lc}
\hline $\begin{array}{l}\text { Freqüência de } \\
\text { pastejo (dias) }\end{array}$ & $\begin{array}{c}\text { Relação folha:haste } \\
\text { Leaf:stem ratio }\end{array}$ \\
\hline 28 & $1,32^{\mathrm{a}}$ \\
38 & $1,16^{\mathrm{ab}}$ \\
48 & $0,99^{\mathrm{b}}$ \\
\hline${ }^{1}$ Médias seguidas da mesma letra não diferem significativamente \\
pelo teste LSD ( $\mathrm{P}<0,05)$. \\
2 Os dados foram analisados segundo a transformação logarítmica \\
\multicolumn{2}{c}{ decimal. As médias foram re-transformadas. } \\
${ }^{1}$ Means followed by the same letters do not differ significantly by the $L S D$ test \\
${ }^{2}(P<.05)$.
\end{tabular}

maio, sendo intermediário em janeiro/fevereiro e fevereiro/abril. A baixa porcentagem de folhas em abril/maio é decorrente da predominância de perfilhos reprodutivos nessa época do ano.

Na cultivar Tanzânia houve interação $(P<0,05)$ entre freqüência de pastejo e época do ano para a variável relação $\mathrm{F}: \mathrm{H}$ (Tabela 5). Em novembro/dezembro, a participação das folhas aumentou com o intervalo de pastejos, o que pode ser atribuído à reduzida taxa de alongamento das hastes aliada à elevada taxa de alongamento das folhas nesse período, ou seja, a extensão do período de descanso favoreceu mais o acúmulo de folhas que de hastes (SANTOS, 1997). Em janeiro/fevereiro não houve efeito da freqüência de pastejo sobre a relação folha:haste. Em fevereiro/abril a relação folha:haste reduziu quando se passou de 28 para 38 dias de intervalo de pastejos, porém não houve diferença entre as freqüências de 38 e 48 dias. Na época do florescimento (abril/maio), a relação folha:haste diminuiu com a redução da freqüência de pastejo.

ANDRADE (1987) observou que as hastes apresentavam menor teor de proteína que as folhas nas cultivares Tobiatã e Colonião. SINGH (1995) encontrou correlação positiva entre a relação F:H e a digestibilidade de matéria seca para diversos genótipos de P. maximum; além disso, elevada proporção de hastes pode limitar o consumo dos animais (FLORES et al., 1993).

PARSONS e PENNING (1988) determinaram que o melhor balanço entre fotossíntese, produção e senescência é obtido quando a forragem é colhida ao atingir a máxima taxa de acúmulo líquido. No entanto, quando a taxa de acúmulo se mantém elevada, mas a presença das hastes começa a limitar o consumo de forragem, o manejo deve ser direcionado para reduzir a participação das hastes na produção.

Para PINTO et al. (1994), o valor limite para a relação $\mathrm{F}: \mathrm{H}$ seria 1,0 . Tanto para o Tanzânia como para o Mombaça, os maiores valores de relação F:H foram obtidos em novembro/dezembro, porém estes nunca foram superiores a 1,8. Entre janeiro, fevereiro e março, a participação das hastes aumentou e a relação $\mathrm{F}: \mathrm{H}$ ficou entre 1,02 e 1,18 (exceto para a cultivar Tanzânia com 28 dias). Já em abril/maio, os valores foram iguais ou inferiores a 1,0 .

Tabela 6 - Relação folha:haste da cultivar Mombaça nas quatro épocas do ano ${ }^{1,2}$

Table 6 - Leaf:stem ratio of Mombaça over the four periods 1, 2 Época do ano Relação folha:haste

Period

$14 / 11$ a $31 / 12 / 1995$ Leaf:stem ratio

$1 / 1$ a $17 / 2 / 1996$

$1,87^{\mathrm{a}}$

$18 / 2$ a $5 / 4 / 1996$

$1,14^{\mathrm{b}}$

$6 / 4$ a 23/5/1996

$1,13^{\mathrm{b}}$

$0,71^{\mathrm{c}}$

${ }^{1}$ Médias seguidas da mesma letra não diferem significativamente pelo teste LSD $(P<0,05)$

2 Os dados foram analisados segundo a transformação logarítmica decimal. As médias foram re-transformadas.

${ }^{1}$ Means followed by the same letters do not differ significantly by the LSD test $(P<.05)$.

2 Herbage mass data are $\log _{10}$ transformed. Means were back transformed.

Tabela 7 - Efeito da freqüência de pastejo e da época do ano na relação folha:haste da cultivar Tanzânia ${ }^{1,2}$

Table 7 - Effects of grazing frequency and periods on the leaf:stem ratio of Tanzânia 1,2

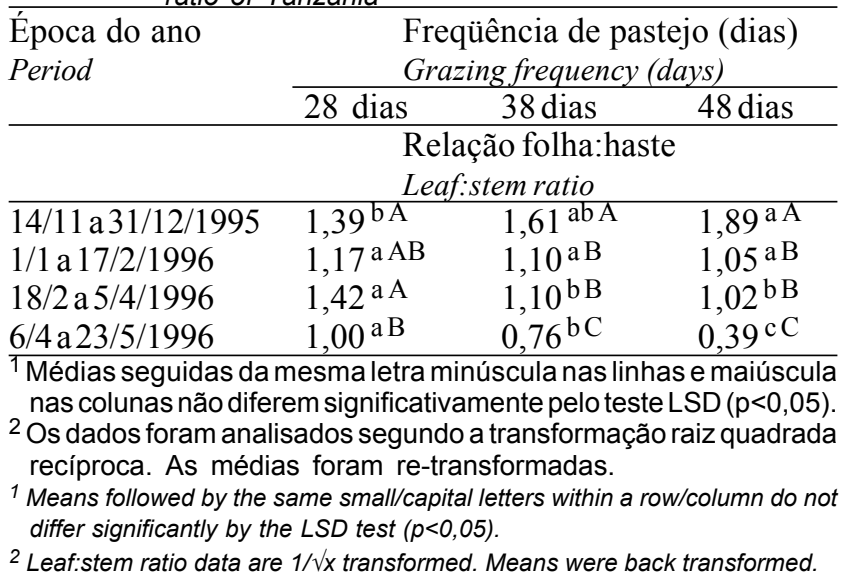


Apesar de o valor limite sugerido por PINTO et al. (1994) ser arbitrário, a relação $\mathrm{F}: H$ das cultivares Tanzânia e Mombaça, nas condições estudadas, foi inegavelmente baixa, principalmente na fase reprodutiva (abril/maio). Isso mostra que é necessário buscar formas de controlar a participação das hastes na produção. As elevadas relações $\mathrm{F}: \mathrm{H}$ com 28 dias de intervalo de pastejos, aliadas à ausência de efeito do intervalo de pastejos sobre a taxa de acúmulo de forragem, sugerem a possibilidade de se aumentar a freqüência de pastejo como alternativa para o controle do crescimento das hastes. Desta forma, constata-se que, tanto para o Mombaça quanto para o Tanzânia, os pastejos devem ser realizados a intervalos iguais ou menores que 28 dias no período de abril/maio (florescimento).

\section{Conclusões}

Apesar de a massa de forragem ser maior quando a freqüência de pastejo é menor, não se observou efeito sobre a taxa de acúmulo de MS, tanto na cultivar Tanzânia quanto na Mombaça. Para as duas cultivares, a taxa de acúmulo de MS foi maior em janeiro/fevereiro, constatando-se que nessa época devam ser adotadas freqüências de pastejo ou lotações animais mais elevadas.

O efeito do intervalo de pastejos sobre a relação F:H do Tanzânia depende da época de avaliação. No Mombaça, a participação das folhas diminuiu com o aumento do intervalo de pastejos. De modo geral, a relação $\mathrm{F}: \mathrm{H}$ das duas cultivares foi baixa, mostrando que essa característica deve ser considerada para a determinação do manejo. Durante a fase reprodutiva (abril/maio), sugere-se aumentar a freqüência de pastejo (pastejos a cada 28 dias ou menos), a fim de tentar controlar o desenvolvimento das hastes.

\section{Referências Bibliográficas}

ANDRADE, J.B. Estudo comparativo de 3 capins da espécie Panicum maximum Jacq. (colonião, tobiatã e K-187-B): Piracicaba, SP: ESALQ, 1987. Dissertação (Mestrado em Agronomia) - Escola Superior de Agricultura "Luiz de Queiroz"/USP, 1987.

CECATO, U., FAVORETTO, V., MALHEIROS, E.B. 1994. Influência da freqüência de corte de níveis e formas de aplicação de nitrogênio sobre a produção e a taxa de crescimento do capim-aruana. R. UNIMAR, 16:203-216 (sup.).

CECATO, U., MARCO, A.A.F.B., SAKAGUTI, E.S. et al. Avaliação de cultivares de Panicum maximum Jacq. In: REUNIÃO ANUAL DA SOCIEDADE BRASILEIRA DE
ZOOTECNIA, 33, 1996, Fortaleza, CE. Anais... Fortaleza: SBZ, 1996, p.109-111.

CORSI, M. Effects of nitrogen rates and harvesting intervals on dry matter productivity, tillering and quality of the tropical grass Panicum maximum (Jacq.). Ohio, EUA: Ohio State University, 1984, 125 p. Tese (PhD).

Costa, C., FAvoretto, V., MALheiros, E.B. 1992. Estudo da variação na estrutura da vegetação de duas cultivares de Panicum maximum Jacq. (colonião e tobiatã) submetidas a diferentes tipos de manejo. 1. Produção e densidade de perfilhos e de matéria seca. Pesq. Agropec. Bras., 27(1):131-142.

FLORES, E.R., LACA, E.A., GRIGGS, T.C. et al. 1993. Sward height and vertical morphological differentiation determine cattle bite dimensions. Agronomy J., 85(3):527-532.

HASSAN, W.E.W., PHIPPS, R.H., OWEN, E. 1990. Dry matter yield and nutritive value of improved pastures species in Malaysia. Trop. Agric., 67(4):303-308.

JANK, L., SAVIDAN, Y., SOUZA, M.T. et al. 1994. Avaliação do germoplasma de Panicum maximum introduzido da África. 1. Produção forrageira. R. Soc. Bras. Zootec., 23(3):433-440.

JANK, L. Melhoramento e seleção de variedades de Panicum maximum . In: SIMPÓSIO SOBRE MANEJO DA PASTAGEM, 12, Piracicaba, 1995. Anais... Piracicaba: FEALQ, 1995. p.21-58.

PARSONS, A.J., PENNING, P.D. 1988. The effect of duration of regrowth on photosynthesis, leaf death and the average rate of growth in a rotationally grazed sward. Grass and Forage Sci., 43:15-27.

PINTO, J.C., GOMIDE, J.A., MAESTRI, M. 1994. Produção de matéria seca e relação folha:caule de gramíneas forrageiras tropicais, cultivadas em vasos, com duas doses de nitrogênio. R. Soc. Bras. Zootec., 23(3):313-326.

SANTOS, P.M. Estudo de algumas características agronômicas de Panicum maximum (Jacq.) cvs. Tanzânia e Mombaça para estabelecer seu manejo. Piracicaba, 1997. Dissertação (Mestrado em Zootecnia) - Escola Superior de Agricultura "Luiz de Queiroz"/Universidade de São Paulo, 1997.

SAS INSTITUTE INC. SAS User's guide: Statistics Version, 5.ed. Cary, NC: SAS Institute Inc., 1985, 956 p.

SAVIDAN, Y.H., JANK, L., COSTA, J.C.G. 1990. Registro de 25 acessos selecionados de Panicum maximum, Campo Grande, EMBRAPA-CNPGC. 68 p. (EMBRAPA-CNPGC, Documentos $\left.n^{\circ} 44\right)$.

SILVA, D. S., GOMIDE, J.A., QUEIROZ, A.C. 1994. Pressão de pastejo em pastagem de capim-elefante anão (Pennisetum purpureum, Schum C.V. Mott). 2. Efeito sobre o valor nutritivo, consumo de pasto e produção de leite. R. Soc. Bras. Zootec., 23(3):453-465.

SINGH, D.K. 1995. Effect of cutting management on yield and quality of different selections of guinea grass (Panicum maximum (Jacq.) L.) in a humid subtropical environment. Trop. Agric., 72(3):181-187.

VILLAREAL, M. 1994. Valor nutritivo de gramíneas y leguminosas forrajeras en San Carlos, Costa Rica. Pasturas Tropicales, 16(1):27-31.
Recebido em: 16/10/97

Aceito em: 20/10/98 